WM-06-105

\title{
Top Pair Production in Randall-Sundrum Models
}

\author{
Erin De Pree ${ }^{1}$ and Marc Sher ${ }^{2}$ \\ Particle Theory Group \\ Department of Physics \\ College of William and Mary, Williamsburg, VA 23187, USA
}

\begin{abstract}
If the lowest lying Kaluza-Klein states in Randall-Sundrum (RS1) models have masses in the $10-100 \mathrm{TeV}$ range, direct production of these states at the LHC or ILC is impossible, and electroweak precision measurements may not be sufficiently sensitive. We address the possibility that high-precision measurements of top pair production at the ILC may provide the first evidence of these states. We consider RS1 models with fermions on and off the brane, with bulk left and right handed mass terms, discuss brane kinetic terms and calculate corrections to top pair production in these models.
\end{abstract}

\footnotetext{
${ }^{1}$ Email: ekdepr@wm.edu

${ }^{2}$ Email: sher@physics.wm.edu
} 


\section{Introduction}

For the past quarter of a century, two of the most promising solutions to the gauge hierarchy problem have been supersymmetry and technicolor. These extensions of the standard model have provided some of the primary motivations for the LHC and the ILC, and have provided a rich framework for studying beyond-the-standard-model phenomenology.

An alternative approach was provided several years ago by the Randall-Sundrum (RS1) model [1]. In this model, spacetime is five-dimensional, with one dimension compactified on an $S_{1} / Z_{2}$ orbifold. The five-dimensional bulk geometry is a slice of anti-de Sitter $\left(A d S_{5}\right)$ space. At the fixed points of the orbifold (at $y=0, \pi R$ ), the slice is bounded by 3 -branes of equal and opposite tension. The brane at $y=0$ is referred to as the Planck brane, while the brane at $y=\pi R$ is referred to as the $\mathrm{TeV}$ brane. The curvature scale, $k$, and the length of the $A d S_{5}$ slice, $\pi R$, are expected to be of the order of the Planck mass, $M_{P}$ and its inverse, respectively. The geometry then induces a effective scale on the $\mathrm{TeV}$ brane of the order of $M_{P} e^{-\pi k R}$. For $k R \simeq 11$, which is not particularly "fine-tuned", this scale is of the order of a TeV. If the Higgs field(s) live on the $\mathrm{TeV}$ brane, then the electroweak scale is naturally generated. Thus, the hierarchy problem is solved. Several very nice reviews of the model, as well as many of the issues discussed in the rest of this section, can be found in Ref. 2]

In the original model, only gravity propagated in the bulk and the standard model fields were confined to the $\mathrm{TeV}$ brane. Nonetheless, this leads to interesting collider effects from Kaluza-Klein (KK) graviton exchange [3]. It was realized at an early stage that a much richer phenomenology would arise if one allowed some of the standard model fields to propagate in the bulk.

Initially, the effects of gauge bosons in the bulk (with the Higgs field and fermions still confined to the $\mathrm{TeV}$ brane) were considered [4, 5]. In this model, the couplings of the fermions to the KK excitations of the gauge bosons are enhanced relative to the couplings to the zero- 
mode gauge bosons by a factor of $\sqrt{2 \pi k R} \simeq 8$.4. These large couplings cause serious constraints [6. 7. 8] from precision electroweak measurements, with bounds ranging from $10-25 \mathrm{TeV}$ on the mass of the lowest lying KK excitation of the gauge bosons. Such a high mass would be beyond the reach of the LHC, and would also reintroduce the hierarchy problem (although at a much smaller level of fine-tuning).

One method of relaxing these constraints, with fermions still on the $\mathrm{TeV}$ brane, is to include brane-localized kinetic terms for the gauge fields. These terms should be present in general [9]. Their effects on couplings and masses were shown to be substantial in flat space [10], and an analysis [1] in the RS model showed that the lower bound on the lightest KK excitation mass could be substantially smaller.

An alternative approach to relaxing the constraints is to allow fermions to propagate in the bulk. This also gives the exciting possibility of explaining the large fermion mass hierarchies. With fermions in the bulk, the bounds from electroweak precision data were somewhat ameliorated [7, 12, 13, 14, 15, 16, 17]. In addition, since fermions are in the bulk, the couplings of the fermions to the Higgs boson (which remains on the $\mathrm{TeV}$ brane) can be substantially suppressed by the geometric warp factor [12, 18, 19, 20, For fermions near the TeV brane, the suppression is small, but for fermions far from the $\mathrm{TeV}$ brane, the suppression can be exponential, leading to large fermion mass hierarchies. The observed fermion mass hierarchy then becomes a matter of fermion geography. Huber [20] has shown explicitly how simple parameters of $O(1)$ can lead to the observed fermion mass hierarchy and mixings.

As shown by Agashe, et al. [21], the model still had large contributions to the T parameter in electroweak radiative corrections, forcing the KK scale to still be out of reach of the LHC. It also had large contributions to $Z \rightarrow \bar{b} b$. The reason is that the large top quark mass forces the top quark to be near the $\mathrm{TeV}$ brane, so that it can interact strongly with the Higgs. But since the left-handed top is paired with the left-handed bottom, the left-handed bottom will have to be near the $\mathrm{TeV}$ brane, and that leads to larger corrections to the $Z \rightarrow \bar{b} b$ rate. They 
showed that imposing a custodial isospin symmetry in the bulk (by enlarging the gauge group to $\left.S U(2)_{L} \times S U(2)_{R} \times U(1)_{B-L}\right)$ solves both of these problems, and allows the lowest lying KK states to have masses as low as a few $\mathrm{TeV}$, within range of the LHC. These models are attractive in that the custodial isospin gauge symmetry of the bulk can be dual, through the AdS/CFT correspondence, to a global isospin symmetry of the CFT.

There are other alternatives. Hewett, Petriello and Rizzo [22] consider putting the first two families in the bulk and the third on the brane, and alleviate these problems. This paper was the first to consider top pair production in Randall-Sundrum models at a linear collider, although it was in the context of the model with the third generation on the brane and used a common mass parameter for the other fermions. More recently, Carena et al. [17] show that brane kinetic terms for the fermions can also give good fits for relatively light KK masses. An introduction to brane kinetic terms can be found in Ref. [23. A summary of many of these issues, including flavor changing neutral currents, can be found in Ref. [24], where it is pointed out that the KK mass scale could be lowered to the few $\mathrm{TeV}$ mass scale without problems with precision electroweak data.

Our approach in this paper is somewhat different. We will not attempt to find ways to lower the KK masses to the range of the LHC, but will consider the possibility that these masses are in the $10-100 \mathrm{TeV}$ range. In this case, they will be out of reach of the LHC and ILC, and (except possibly in the lower end of the range for some models) will be insensitive to electroweak precision measurements (and any sensitivity can be eliminated with one of the techniques discussed above). Of course, there will be a hierarchy problem, although substantially less of a problem than in standard grand unified theories, and we will not address that issue. In this scenario, what would the first experimental evidence be? Since the top quark is close to the $\mathrm{TeV}$ brane, effects of KK states on top pair production would be the most pronounced, and thus could be the first signature (more likely at the ILC, where higher precision measurements can be made). In this work, we study top pair production in a variety of RS models, and determine 
the reach of KK masses expected at the ILC.

In Section 2, the RS models are presented. In Sections 3, we consider only the effects of KK gauge bosons, ignoring KK fermions. In Section 4, the effects of KK fermions and of brane kinetic terms are considered. Finally Section 5 contains our conclusions.

\section{The Models}

The metric of the Randall-Sundrum model [1] is given by

$$
d s^{2}=e^{-2 \sigma(y)} \eta_{\mu \nu} d x^{\mu} d x^{\nu}-d y^{2}
$$

where $\sigma(y)=k|y|, k$ is related to the curvature of the AdS space, $\eta_{\mu \nu}$ is the flat-space metric, and $y$ is the fifth coordinate. The fifth dimension is compactified on an $S^{1} / Z_{2}$ orbifold bounded by branes at the fixed points $y=0$ and $y=\pi R$. In this section, we present the masses and couplings of gauge bosons and fermions, when they propagate in the bulk. More detailed derivations of these results can be found in references cited in the last section.

The equation of motion for a bulk gauge field is given by [4, 5, 7, 15, 20.

$$
\frac{1}{\sqrt{-G}} \partial_{M}\left(\sqrt{-G} G^{M N} G^{R S} F_{N S}\right)-M_{A}^{2} G^{R S} A_{S}=0
$$

where $M_{A}$ arises from spontaneous symmetry breaking, $G^{M N}$ is the above metric and $\sqrt{-G}=$ $e^{-2 \sigma}$. This can be rewritten as

$$
\left[e^{2 \sigma} \eta_{\rho \nu} \partial^{\rho} \partial^{\nu}+e^{2 \sigma} \partial_{5}\left(e^{-2 \sigma} \partial_{5}\right)-M_{A}^{2}\right] A\left(x_{\mu}, y\right)=0
$$

The Higgs field is localized on the $\mathrm{TeV}$ brane, and thus $M_{A}^{2}=\frac{1}{2} g_{5}^{2} v^{2} \delta(y-\pi R)$. The vacuum expectation value is of the order of the Planck mass.

Decomposing the gauge field (using the gauge $A_{5}=\partial_{\mu} A^{\mu}=0$ ), one has

$$
A\left(x_{\mu}, y\right)=\frac{1}{\sqrt{2 \pi R}} \sum_{n=0}^{\infty} A^{(n)}\left(x_{\mu}\right) f_{n}^{A}(y)
$$


where the orthogonality condition is

$$
\frac{1}{2 \pi R} \int_{-\pi R}^{\pi R} d y f_{n}^{A}(y) f_{m}^{A}(y)=\delta_{m n}
$$

Plugging the decomposition into the equation of motion, one can solve the equation and find [4, 5, 20]

$$
f_{n}^{A}(y)=\frac{e^{\sigma}}{N}\left[J_{1}\left(\frac{m_{n}}{k} e^{\sigma}\right)+b_{1}\left(m_{n}\right) Y_{1}\left(\frac{m_{n}}{k}\right)\right]
$$

The values of $m_{n}$ and $b$ are given by the boundary conditions, and $N$ by the normalization condition. Note that the mass term does not enter into this equation; it will only affect the boundary conditions at the $\mathrm{TeV}$ brane. Imposing these conditions gives the zero-mode mass $20]$

$$
m_{0}^{2}=g_{5}^{2} v^{2} e^{-2 \pi k R}\left(1+\mathcal{O}\left(g_{5}^{2} v^{2} e^{-2 \pi k R} / M_{1}^{2}\right)\right)
$$

where $M_{1}$ is the KK scale. Note that a gauge hierarchy naturally appears. The higher order correction causes a tree-level shift in the $\mathrm{W}$ and $\mathrm{Z}$ masses, affecting electroweak precision data if the KK scale is too small, leading to many of the bounds noted in the previous section. The masses of the KK-excitations of the gauge bosons are related to zeroes of the Bessel functions. One can add brane kinetic terms for the gauge bosons, as will be discussed in Section 4 .

If the fermions are on the $\mathrm{TeV}$ brane, then, as shown in Refs. [4, 5], their couplings to the gauge bosons are of the form

$$
\mathcal{L}=-g \bar{\psi} \gamma^{\mu}\left(A_{\mu}^{(0)}+\sqrt{2 \pi k R} \sum_{n=1}^{\infty} A_{\mu}^{(n)}\right) \psi
$$

which gives an enhancement of $\sqrt{2 \pi k R} \simeq 8.4$ in the coupling. This changes substantially if the fermions are in the bulk.

When fermions are in the bulk [12, 18, 20], they can have two possible transformation properties under the orbifold $Z_{2}$ symmetry: $\psi= \pm \gamma_{5} \psi$. As a result, $\bar{\psi} \psi$ is odd under the $Z_{2}$, and thus the Dirac mass term must originate from coupling to a $Z_{2}$ odd scalar field. This mass 
term can then be written as $m_{\psi}=c \frac{d \sigma}{d y}$, where $\sigma=k|y|$. As we will see shortly, the parameter $c$ will be crucial in determining the properties of the fermions.

As before, one can expand the fields and determine the wavefunctions and masses of the fermions. One expands

$$
\psi\left(x^{\mu}, y\right)=\frac{1}{2 \pi R} \sum_{n=0}^{\infty} \psi^{n}\left(x^{\mu}\right) e^{2 \sigma} f_{n}(y)
$$

where the normalization condition is

$$
\frac{1}{2 \pi R} \int_{-\pi R}^{\pi R} d y e^{\sigma} f_{m}(y) f_{n}(y)=\delta_{m n}
$$

and the factor of $e^{2 \sigma}$ comes from the spin connection.

Plugging into the Dirac equation, one finds the zero mode wave function is simply (we suppress flavor indices and neglect flavor mixing)

$$
f_{0}(y)=\frac{e^{-c \sigma}}{N_{0}}
$$

and the KK-fermion wave functions are

$$
f_{n}(y)=\frac{e^{\sigma / 2}}{N_{n}}\left[J_{\alpha}\left(\frac{m_{n}}{k} e^{\sigma}\right)+b_{\alpha}\left(m_{n}\right) Y_{\alpha}\left(\frac{m_{n}}{k}\right)\right]
$$

where $\alpha=\left|c \pm \frac{1}{2}\right|$ for $\psi_{L, R}$. The masses and $b_{\alpha}$ are given by the boundary conditions.

The zero-mode wave function is sufficiently simple that the normalization constant $N_{o}$ can be determined easily to be

$$
N_{o}^{2}=\frac{e^{2 \pi k R(1 / 2-c)}-1}{2 \pi k R(1 / 2-c)}
$$

From this, one can see that if $c>1 / 2$, the zero mode fermions will be localized near the Planck $(y=0)$ brane, while for $c<1 / 2$, they will be localized near the $\mathrm{TeV}(y=\pi R)$ brane.

The zero modes acquire mass through coupling to the Higgs field on the TeV brane (here, we include flavor indices)

$$
m_{i j}=\int_{-\pi R}^{\pi R} \frac{d y}{2 \pi R} \lambda_{i j}^{5}\langle H(y)\rangle f_{0 i L}(y) f_{0 j R}(y)
$$


and using $\langle H(y)\rangle=v \delta(y-\pi R) / k$, one finds

$$
m_{i j}=\frac{\lambda_{i j}^{4} v}{\pi k R} f_{0 i L}(\pi R) f_{0 j R}(\pi R)
$$

where the dimensionless 4-D coupling $\lambda_{i j}^{4}=\lambda_{i j}^{5} \sqrt{k}$.

This demonstrates how a huge fermion mass hierarchy can arise. For $c<1 / 2$, the wave function $f_{0}(\pi R)$ varies as $\sqrt{1-2 c}$, but for $c>1 / 2$ varies as $e^{-c \pi k R}$. Since $\pi k R \simeq 35$, this exponential suppression can lead to a hierarchy. Huber [20] shows explicitly how mild variations in $c$ can lead to the observed mass spectrum, and can also lead to reasonable flavor mixing.

The couplings between gauge bosons and fermions come from the 5-D term

$$
\int d^{4} x d y \sqrt{-G} g_{5} \bar{\psi}(x, y) i \gamma^{\mu} A_{\mu}(x, y) \psi(x, y)
$$

which induces $4 \mathrm{D}$-couplings

$$
g_{i j n}=\frac{g_{5}}{(2 \pi R)^{3 / 2}} \int_{-\pi R}^{\pi R} e^{\sigma} f_{i}(y) f_{j}(y) f_{n}^{A}(y) d y
$$

From this, we can now determine all gauge-boson couplings to fermions.

Note that for a zero-mode massless gauge boson, $f_{0}^{A}=1$, and the result just gives the normalization condition, giving $g_{i j 0}=\delta_{i j} g_{5} / \sqrt{2 \pi R}$, thus fermion couplings to the zero-mode are KK-level conserving.

For our calculation, we will need the coupling of a KK-gauge boson to zero-mode fermions, which is then ${ }^{3}$

$$
g^{(n)}=g\left(\frac{1-2 c}{e^{(1-2 c) \pi k R}-1}\right) \frac{k}{N_{0}} \int_{0}^{\pi R} d y e^{\sigma} e^{(1-2 c) \sigma}\left[J_{1}\left(\frac{m_{n}}{k} e^{\sigma}\right)+b_{1}\left(m_{n}\right) Y_{1}\left(\frac{m_{n}}{k}\right)\right] .
$$

These are plotted in Ref. [12 for $n=1,2,3$ as a function of $c$. For $c$ large and negative (so the fermion is very close to the $\mathrm{TeV}$ brane), the coupling ratio reaches $\sqrt{2 \pi k R} \simeq 8.4$, as discussed earlier. As $c$ increases, they become smaller, vanishing in the conformal limit $c=1 / 2$, and then reach a constant value of approximately -0.2 for $c>1 / 2$.

\footnotetext{
${ }^{3}$ In Ref. [12], the first factor of $e^{\sigma}$ in the integral is missing-this is entirely typographical and does not affect their results.
} 
This scenario is very attractive, due to the manner in which the fermion mass hierarchy naturally arises. We can see that fermions near the $\mathrm{TeV}$ brane couple more strongly than those away from the $\mathrm{TeV}$ brane. Since the top quark is closest to the $\mathrm{TeV}$ brane, one expects the biggest effects to arise in top-quark processes, and if the KK-scale is much larger than $10 \mathrm{TeV}$, these processes may be the first signature.

We now turn to top pair production, and first consider only the effect of KK-gauge bosons. Note that in the absence of brane kinetic terms, the masses of the KK-fermions (for a given value of the Dirac mass term) are related to those of KK-gauge bosons (through zeroes of Bessel functions), and such a consideration is not realistic. But since brane kinetic terms can decouple the masses, such a separation is consistent. Following the discussion of the effects of KK-gauge bosons, we will turn to those of KK-fermions.

\section{Effects of KK Gauge Bosons}

\subsection{Fermions on the brane}

As discussed in the previous section, if all of the standard model fermions are on the brane, then their couplings to the KK-gauge bosons are enhanced by a factor of $\sqrt{2 \pi k R} \sim 8.4$. This will lead to substantial corrections to fermion pair production through the diagrams of Fig. 1. In this diagram, we neglect the $\mathrm{n}=1$ weak mixing angle, which is defined as the rotation angle between the hypercharge and $S U(2)$ gauge bosons and their mass eigenstates. The reason for

this is that mixing is due to electroweak symmetry breaking, and the scale of the KK-gauge boson masses is much, much larger. This is similar to the case of universal extra dimensions 25] in which the weak mixing angle for the $n=1$ states was shown to be $O(0.01)$.

The corrections to the top pair production cross section can be easily calculated for the exchange of the $n=1 \mathrm{KK}$-gauge bosons. The result is given in Fig. 2 for $\sqrt{s}=0.5,1.0,1.5$ $\mathrm{TeV}$. The expected sensitivity of the ILC is approximately one percent, and thus the ILC will be able to probe masses up to $120 \mathrm{TeV}$ (for $\sqrt{s}=1.0 \mathrm{TeV}$ ). Note that the interference is 


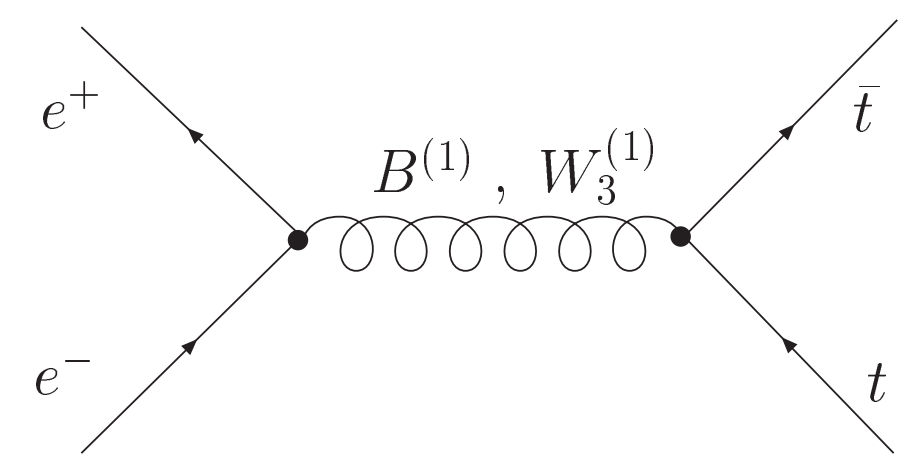

Figure 1: Tree-level diagrams affecting top pair production. The exchanged gauge bosons are the $\mathrm{KK}-W_{3}$ and KK-B.

destructive. The sensitivity to high mass scales should not be surprising, since one expects the change in the cross section to be approximately $2 \times(8.4)^{2} \times \frac{s}{M_{K K}^{2}}$, and a one percent sensitivity for $\sqrt{s}=1 \mathrm{TeV}$ gives a bound on $M_{K K}$ of $120 \mathrm{TeV}$.

One can also have the $n=2,3, \ldots$ KK-gauge bosons exchanged. In universal extra dimensions, the KK-gauge boson masses vary linearly with $n$, and thus one would multiply the result by $\sum_{n=1}^{\infty} \frac{1}{n^{2}} \sim 1.6$. In the Randall-Sundrum case, one must sum over the zeroes of Bessel functions. Doing this numerically, one also gets approximately an enhancement of 1.6. This would increase the bound by approximately 30 percent, if the model isn't cut off at higher scales. Thus, we find sensitivity to masses up to $150 \mathrm{TeV}$.

Note that there is nothing special about the top quark in this calculation-similar results would occur for production of any fermion pair, including muons. Thus, one could obtain sensitivity to even greater mass scales looking at pair production of other fermions.

One could ask about the reliability of perturbation theory. Because of the enhancement, the effective coupling constants of the weak gauge bosons at the $\mathrm{TeV}$ scale are $(8.4)^{2}\left(\frac{\alpha_{w}}{4 \pi}\right) \sim .20$. Depending on coefficients, there could be significant higher order corrections.

If the fermions are not on the brane, then the electron coupling to the KK-gauge bosons will be much weaker since the electron is further away from the $\mathrm{TeV}$ brane. Instead of an enhancement factor of 8.4, the coupling decreases [12] by a factor of roughly 5 . This change 


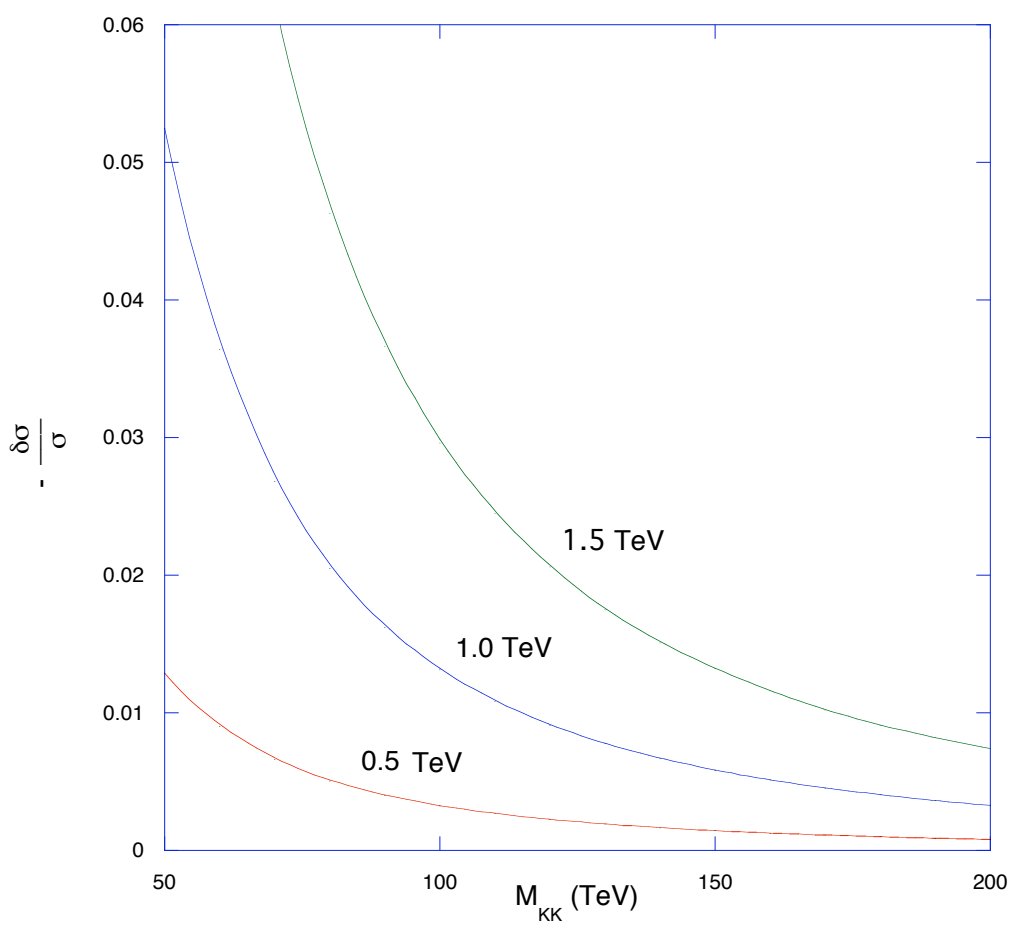

Figure 2: Corrections to the top pair production cross section from the diagrams of Figure 1, as a function of the $\mathrm{n}=1 \mathrm{KK}$-gauge boson mass, for center of mass energies of $0.5,1.0$ and 1.5 $\mathrm{TeV}$. 
alone would reduce the above bound by a factor of $\sqrt{40}$. In addition, the top quark coupling will also be smaller. We consider this bound, as well as other contributions from one-loop corrections, in the next subsection.

\subsection{Fermions off the brane}

As discussed earlier, the scenario in which fermions propagate in the bulk is extremely attractive, in that it provides a simple explanation for the fermion hierarchy. In additon to the tree-level contributions of the last subsection, there are two additional contributions (these are also present in the on-the-wall case, but are substantially smaller than the tree level contributions). One can calculate one-loop diagrams in which the final state top quarks exchange KK-gauge bosons - these can be significant because the gauge bosons can be gluons. The other contribution arises from mixing between the zero mode and KK-gauge bosons. We consider each in turn.

\section{Tree-Level Contributions}

We first consider the same diagrams as in Fig. 1. As noted in the previous paragraph, one expects the bound to be lowered from the on-the-brane case by a factor of at least $\sqrt{40}$, which gives a reach of approximately $25 \mathrm{TeV}$. This will be lowered further since the top quark is not on-the-brane, and so its coupling will be weakened.

In general, the left and right handed top quarks will have different 5-d mass terms, $c_{L}$ and $c_{R}$. This will lead, from Eq. 18, to different enhancements for the different chiralities. If the enhancement of the left handed top quark couplings is $\alpha_{L}$, and that of the right handed top quark couplings is $\alpha_{R}$, one can then determine the cross sections and asymmetries.

Using the notation of Ref. [26] for exchange of a neutral heavy gauge boson $Z^{\prime}$, the differ- 
ential cross section can be written as

$$
\frac{d \sigma_{L}}{d \cos \theta}=\frac{\pi \alpha^{2}}{4 s}\left\{\left|C_{L L}\right|^{2}(1+\cos \theta)^{2}+\left|C_{L R}\right|^{2}(1-\cos \theta)^{2}\right\}
$$

where

$$
C_{i j}=-Q_{f}+\frac{C_{i}^{e} C_{j}^{t}}{c_{w}^{2} s_{w}^{2}} \frac{s}{\left(s-M_{Z}^{2}\right)+i \Gamma_{Z} M_{Z}}+\frac{\left(g_{Z^{\prime}} / g_{Z^{0}}\right)^{2} C_{i}^{e^{\prime}} C_{j}^{t^{\prime}}}{c_{w}^{2} s_{w}^{2}} \frac{s}{\left(s-M_{Z^{\prime}}^{2}\right)+i \Gamma_{Z^{\prime}} M_{Z^{\prime}}}
$$

Here, $C_{i}^{t}$ are the SM $Z^{0}$ couplings and $C_{i}^{t^{\prime}}$ are the $Z^{\prime}$ couplings to the top quark. For righthanded electrons, one substitutes $C_{L L} \rightarrow C_{R R}$ and $C_{L R} \rightarrow C_{R L}$. From this, one finds the unpolarized total cross section is given by

$$
\sigma=\frac{\pi \alpha^{2}}{3 s}\left[\left|C_{L L}\right|^{2}+\left|C_{R L}\right|^{2}+\left|C_{L R}\right|^{2}+\left|C_{R R}\right|^{2}\right]
$$

the forward-backward asymmetry is given by

$$
A_{F B}=\frac{\left[\int_{0}^{1}-\int_{-1}^{0}\right] d \cos \theta \frac{d \sigma}{d \cos \theta}}{\left[\int_{0}^{1}+\int_{-1}^{0}\right] d \cos \theta \frac{d \sigma}{d \cos \theta}}
$$

and the left-right asymmetry is

$$
A_{L R}^{f}=\frac{\sigma\left(e_{L}^{-}\right)-\sigma\left(e_{R}^{-}\right)}{\sigma\left(e_{L}^{-}\right)+\sigma\left(e_{R}^{-}\right)} .
$$

Using these results, we find that the corrections to the cross section, forward-backward asymmetry and left-right asymmetry (using the expected value [12] of -0.2 for the change in the electron coupling to the KK gauge bosons) are given by

$$
\begin{aligned}
\frac{\delta \sigma}{\sigma} & =\left(0.24 \alpha_{L}+0.14 \alpha_{R}\right) \frac{s}{M_{K K}^{2}} \\
\delta A_{F B} & =\left(-0.04 \alpha_{L}-0.03 \alpha_{R}\right) \frac{s}{M_{K K}^{2}} \\
\delta A_{L R} & =\left(0.26 \alpha_{L}-0.19 \alpha_{R}\right) \frac{s}{M_{K K}^{2}}
\end{aligned}
$$




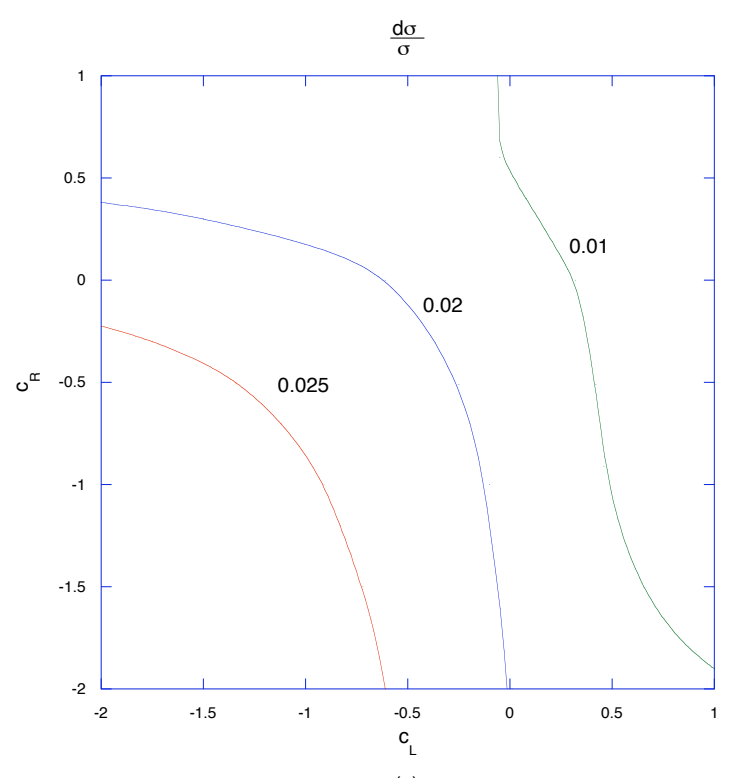

(a)

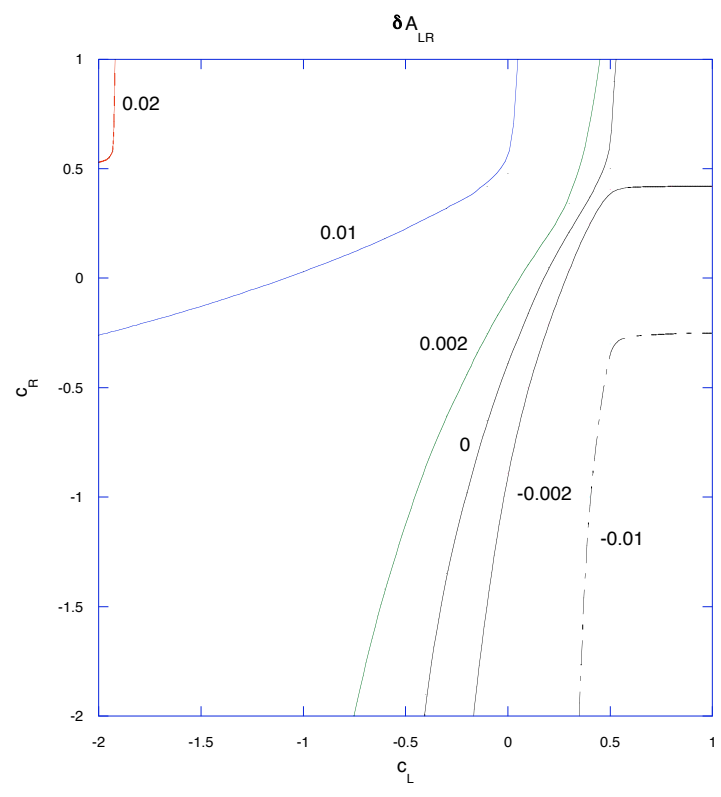

(b)

Figure 3: For different values of $c_{L}$ and $c_{R}$, corrections to the (a) production cross-section and (b) the left-right asymmetry. The contribution to the forward-backward asymmetry is negligible. We have assumed that $M_{K K}=10 \mathrm{TeV}$; the results will scale as $1 / M_{K K}^{2}$.

The result is plotted in Figure 3 as a function of $c_{L}$ and $c_{R}$. Here, we choose $M_{K K}=10$ $\mathrm{TeV}$, the results in all cases scale like the inverse-square of $M_{K K}$. These results are for the $n=1 \mathrm{KK}$ gauge bosons. Including the sum of all KK-modes results in a small change of less than 20 percent (this is less than the sixty percent correction in the last subsection since for some values of the mass term, the couplings of higher modes can be negative).

Depending on how precisely the luminosity at an ILC can be determined, a one-percent measurement of the cross-section is possible, and thus a reach of $10 \mathrm{TeV}$ for much of parameter space can be obtained (and a reach of $15 \mathrm{TeV}$ for some of parameter space is possible). The forward-backward asymmetry is too small to be measurable. The left-right asymmetry is interesting. With a million top pairs expected in several years running, half from left-handed and half from right-handed electrons, assuming $80 \%$ polarization, one could reach a sensitivity of approximately 0.002 for $A_{L R}$, which would also cover most of parameter space, for a $10 \mathrm{TeV}$ KK gauge boson mass, and would cover some of the space even for a $30 \mathrm{TeV}$ mass. It should 


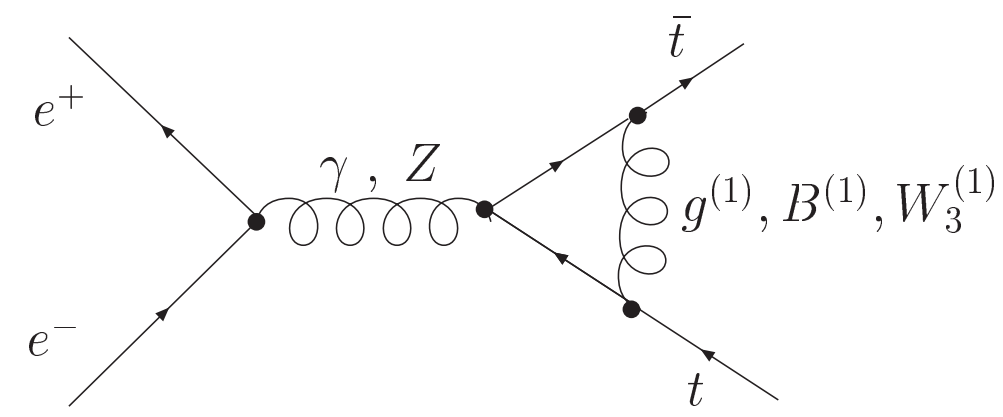

Figure 4: The dominant one-loop diagrams affecting top pair production. The exchanged gauge boson is either a KK-gluon, $\mathrm{KK}-W_{3}$ or a $\mathrm{KK}-\mathrm{B}$. Corrections to the electron vertex are negligible since the electron couplings to the KK-gauge bosons are suppressed. Other diagrams, noted in Appendix A, do not involve KK-gluons and are numerically small.

be noted that the "preferred" range of $c_{L}, c_{R}$, since the right handed top can be much closer to the $\mathrm{TeV}$ brane, is for negative (or near zero) $c_{R}$ and for $c_{L}$ positive (but less than 0.5). A clear signature of the model, which could distinguish it from extra- $\mathrm{Z}$ models, is the absence of a substantial change in the forward-backward asymmetry.

These bounds could perhaps be improved substantially by including the effects of positron beam polarization and of top quark polarization [26], which can increase the bounds by up to a factor of two. This improvement, of course, depends on the design of the ILC.

\section{One-loop Contributions}

We now turn to one-loop corrections to the $\bar{t} t \gamma$ and the $\bar{t} t Z$ vertices. We start with the diagrams in Fig. 4. The exchanged KK-gauge boson can be either a KK-gluon, KK- $W_{3}$, or a KK-B. Of course, one expects the KK-gluon to have the biggest effect; this is the KK-version of the well-known $\frac{\alpha}{\pi}$ correction to the value of $\mathrm{R}$ in hadron production. In fact, we find this to be the case, but present the results for all of the diagrams for completeness.

The most general interactions of the top quark with the $\gamma$ and $Z$, assuming massless initial 
fermions and ignoring the (small) CP-violation, is

$$
\Gamma_{\mu}^{V}\left(q^{2}\right)=-i e\left[\gamma_{\mu}\left(F_{1 V}^{V}\left(q^{2}\right)+\gamma_{5} F_{1 A}^{V}\left(q^{2}\right)\right)+\frac{i \sigma_{\mu \nu} q^{\nu}}{2 m}\left(i F_{2 V}^{V}\left(q^{2}\right)\right)\right]
$$

where $V=\gamma, Z$. As calculated in Ref. [27] and discussed by Baur [28, these coefficients can all be bounded at roughly the one percent level. Baur gives the precise bounds that can be obtained at the ILC. However, the bounds that he lists are from early studies [29], where the integrated luminosity is either 100 or $200 \mathrm{fb}^{-1}$. We are assuming that many years of running at an ILC can yield an integrated luminosity of an inverse attobarn, and thus one can (in the extremely optimistic case of assuming statistical uncertainties only) scale the results by the square-root of the integrated luminosity ratio for interference diagrams, and the fourth-root for direct terms. Positron polarization (50\%) also lowers the limits by $25 \%$, and a center-of-mass energy of $1 \mathrm{TeV}$ also lowers them by a factor of 1.5 [29], compared to the earlier studies which assumed half the center-of-mass energy and no polarization. Including these latter two effects, we take the range of the bounds on the coefficients to be between the values cited by Baur and the optimistic range given with an inverse attobarn luminosity. The ranges of interest are then

$$
\begin{aligned}
& F_{1 V}^{\gamma}: .010-.024 \\
& F_{1 A}^{\gamma}: .003-.006 \\
& F_{2 V}^{\gamma}: .010-.019 \\
& F_{1 V}^{Z}: .003-.006 \\
& F_{1 A}^{Z}: .002-.006 \\
& F_{2 V}^{Z}: .002-.006
\end{aligned}
$$

In principle, one could add the effects of these diagrams to the tree-level contribution, and calculate the resulting cross sections and polarization asymmetries in a unified manner. One could calculate the corrections to the cross section and asymmetries for a given $F$; for example, one can show that the contribution of $F_{1 V}^{Z}$ to $\delta \sigma / \sigma$ is negligible, whereas the contribution of 
$F_{1 A}^{Z}$ is roughly $\delta \sigma / \sigma=2.2 \delta F_{1 A}^{Z}$. However, the tree-level contribution is similar to that of an extra $\mathrm{Z}$ boson for which virtually all studies generally refer to cross sections and asymmetries, while the one-loop contribution involves anomalous $\gamma$ and $Z$ interactions, for which studies generally refer to the above form factors. Furthermore, the sensitivity to changes in the cross section and asymmetries were calculated using different assumptions about the collider than those for the sensitivity to changes in the form factors. Since the detailed specifications of the ILC and its detectors are not yet known, we are simply referring to previous studies and thus keep the contributions separate. A more detailed unified study, including top quark and positron polarization asymmetries would be valuable and could make our results more precise.

The detailed calculations are given in Appendix A. For a given value of $c_{L}$ and $c_{R}$, we can find the enhancements of the couplings of the left and right-handed top quarks, determine the value of $C$ and $\alpha$ in the vertex (see Appendix A), plug into the expressions and determine the effect on the six parameters in Eq. 25, for $q^{2}=s=1 \mathrm{TeV}^{2}$. As in the tree-level case, including higher order terms will increase the mass reach by approximately $20 \%$ - more precision is unnecessary since higher order corrections (such as double KK-gluon exchange) will likely have a bigger effect. The results are plotted in Figure 5, assuming $M_{K K}=5 \mathrm{TeV}$. We see that the most sensitive coefficients are the couplings of the $Z$, for which sensitivities to $M_{K K}=5$ $\mathrm{TeV}$ are reached for most of parameter space. However, we have found that for $M_{K K}=10$ $\mathrm{TeV}$, only a small sliver of parameter space is sensitive. These results are substantially weaker than the results for the tree-level contribution of the last subsection.

\section{Contributions from mixing}

The most detailed discussion of top pair production at a linear collider in the RandallSundrum model was by Agashe, Delgado, May and Sundrum (ADMS) [21], which was recently 

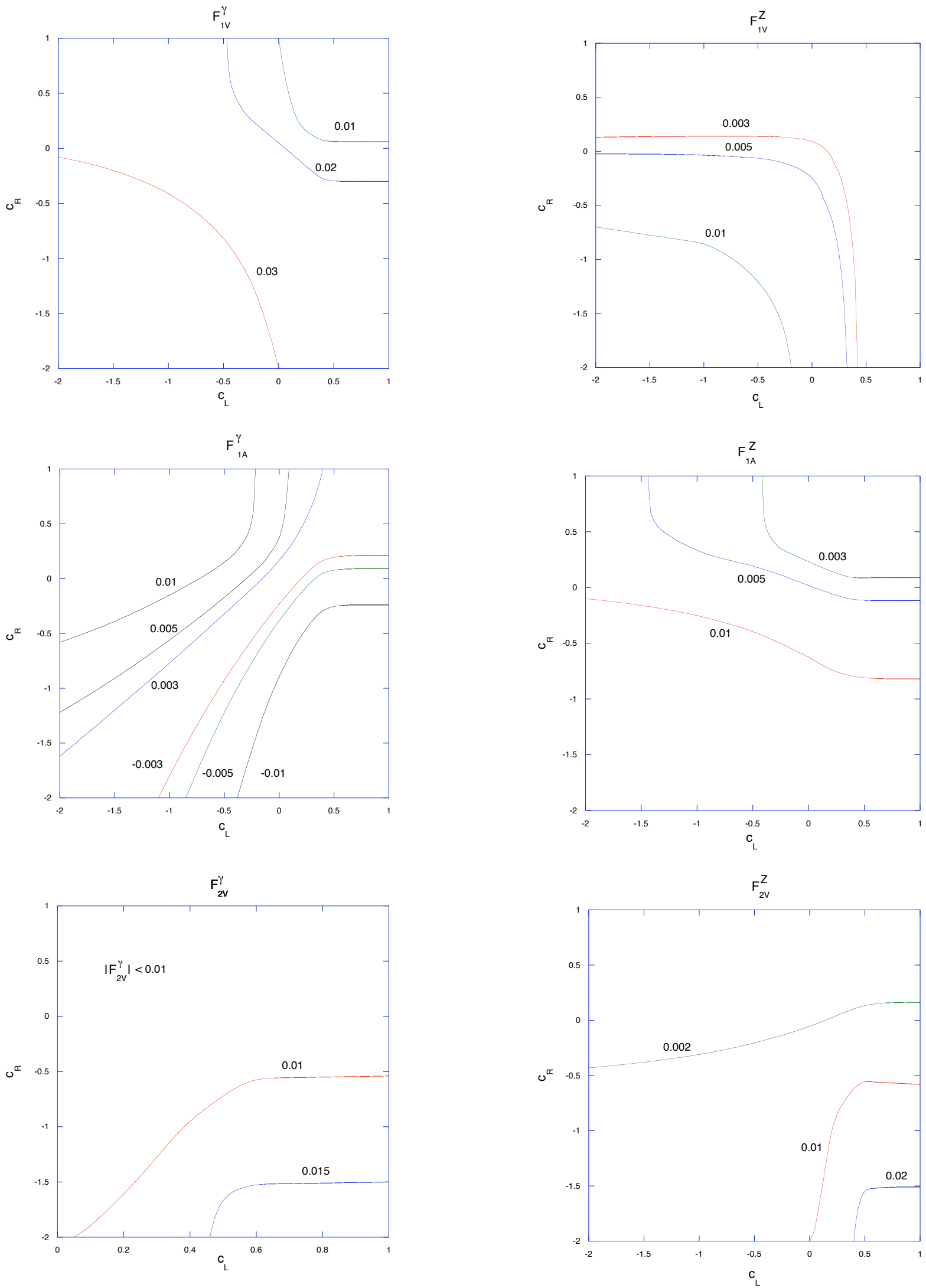

Figure 5: Contributions to the $\gamma$ and $\mathrm{Z}$ form factors as a function of $c_{L}$ and $c_{R}$, for $M_{K K}=5$ $\mathrm{TeV}$. 
summarized by Agashe [30. They discuss the contributions from mixing between the Z-boson and the KK-Z bosons. This mixing occurs from the Higgs vev. The biggest effect is on the right-handed top quark coupling, and they find that

$$
\frac{\delta\left(g_{Z}^{t_{R}}\right)}{g_{Z}^{t_{R}}} \sim \frac{m_{Z}^{2}}{\left(0.41 M_{K K}\right)^{2}} \frac{1-2 c_{R}}{3-2 c_{R}}\left(\frac{-k \pi R}{2}+\frac{5-2 c_{R}}{4\left(3-2 c_{R}\right)}\right)
$$

It is straightforward to convert this into a shift in $F_{1 V}^{Z}$ and $F_{1 A}^{Z}$,

$$
F_{1 V}^{Z}=F_{1 A}^{Z}=-\frac{\tan \theta_{W}}{3} \frac{\delta\left(g_{Z}^{t_{R}}\right)}{g_{Z}^{t_{R}}}
$$

For a KK-gauge boson mass of $5 \mathrm{TeV}$, this gives a result for $F_{1 V}^{Z}$ and $F_{1 A}^{Z}$ which ranges from 0 at $c_{R}=1 / 2$, to 0.002 at $c_{R}=0$, to 0.004 at $c_{R}=-0.2$. We see that the $5 \mathrm{TeV}$ mass scale can barely be reached for the $c_{R}<0$ part of parameter space, and thus could have a greater reach than the one-loop contributions for some of the parameter space. But it is substantially weaker than the tree-level contribution. As we will see in the next section, however, the effects of mixing between the top quark and the KK-top can be substantially larger, and could be competitive with the tree-level contribution.

\section{Effects of KK Fermions and Brane Kinetic Terms}

In our analysis, we have only included the effects of KK-gauge bosons. As noted in Section 2, the masses of the KK-gauge bosons are related to the zeroes of Bessel functions of order 1, while the masses of the KK-fermions are related to zeroes of Bessel functions of order $|c \pm 1 / 2|$. In the absence of brane kinetic terms, the masses of the KK-tops are thus related to those of KK-gauge bosons, and their effects must be considered. In particular, the masses of the left-handed KK-tops [21, 30] are given by $m_{t_{L}^{n}} \sim \pi k e^{-k \pi r_{c}}\left(n-c_{L} / 2\right) \sim 1.28 m_{K K}\left(n-c_{L} / 2\right)$, where $m_{K K}$ is the $n=1 \mathrm{KK}$-gauge boson mass ${ }^{4}$. For $n=1$ and $c_{L}=0.4$, this gives virtually equal $n=1$ KK-top and KK-gauge boson masses.

\footnotetext{
${ }^{4}$ In Ref. [30, there are two typographical errors in Eq. 16 -the factor of $\sqrt{1 / 2-c_{L}}$ should be in the denominator and the factor of 0.78 should be $1 / 0.78$. There are purely typographical and do not affect the results.
} 
Clearly, the results from tree-level KK-gauge boson exchange will not be affected, except for small mixing effects, by KK-top contributions. There will, however, be contributions to the one-loop diagrams of Figure 4, in which the internal top quark lines are replaced by KK-top quark lines. We have calculated the effects of these contributions, and find them to be smaller, in all cases, than the previous results.

A much bigger effect arises from mixing between the top quark and the KK-top quark. This arises from mixing of the zero-mode $t_{R}$ with the KK- $t_{L}$ through the Higgs vev, and is discussed in detail by Agashe [30. Using Eq. 28. Agashe's result can be written as

$$
\delta F_{1 V}^{Z}=F_{1 A}^{Z} \sim \sum_{n} \frac{-1}{2 \sin 2 \theta_{W}}\left(\frac{m_{t}}{m_{t_{L}^{(n)}}}\right)^{2}\left(\frac{1-e^{-2 k \pi R\left(1 / 2-c_{L}\right)}}{1 / 2-c_{L}}\right) .
$$

This is plotted as a function of $c_{L}$ for several masses in Figure 6, where the sum over the KK-modes has been included. The range $c_{L}>0.5$ is exceeedingly disfavored, since the Yukawa coupling of the top quark would then be exponentially suppressed. We see that for $c_{L}=0.4$, a reach of $10 \mathrm{TeV}$ is barely possible, with the optimistic assumptions discussed earlier for the reach of the ILC. For $c_{L}$ very close to 0.5 , however, the reach can exceed that of the tree-level KK-gauge boson exchange.

Thus, mixing can give a reach which can be larger than that of the tree-level KK-gauge boson exchange, but only in the upper end of the $0.4 \leq c_{L} \leq 0.5$ range. Although this seems narrow, it is a particularly interesting range of $c_{L}$. If $c_{L}$ were larger, the Yukawa coupling would be suppressed and the top mass would be too small, and if it were much smaller, there might be dangerous contributions to the $\bar{b} b Z$ vertex. A word of caution is that the large mixing can cause problems with precision electroweak fits, although a custodial SU(2) symmetry or brane kinetic terms can ameliorate the problems (if there is a custodial $\mathrm{SU}(2)$ symmetry, one should include effects of the $Z^{\prime}$ as well). Mixing contributions between the zero-mode $t_{L}$ and the KK- $t_{R}$ are expected to be small since $c_{R}$ is not expected to be in this range. Note that a clear signature of the dominance of mixing would be the equality of the contributions to $F_{1 V}^{Z}$ 


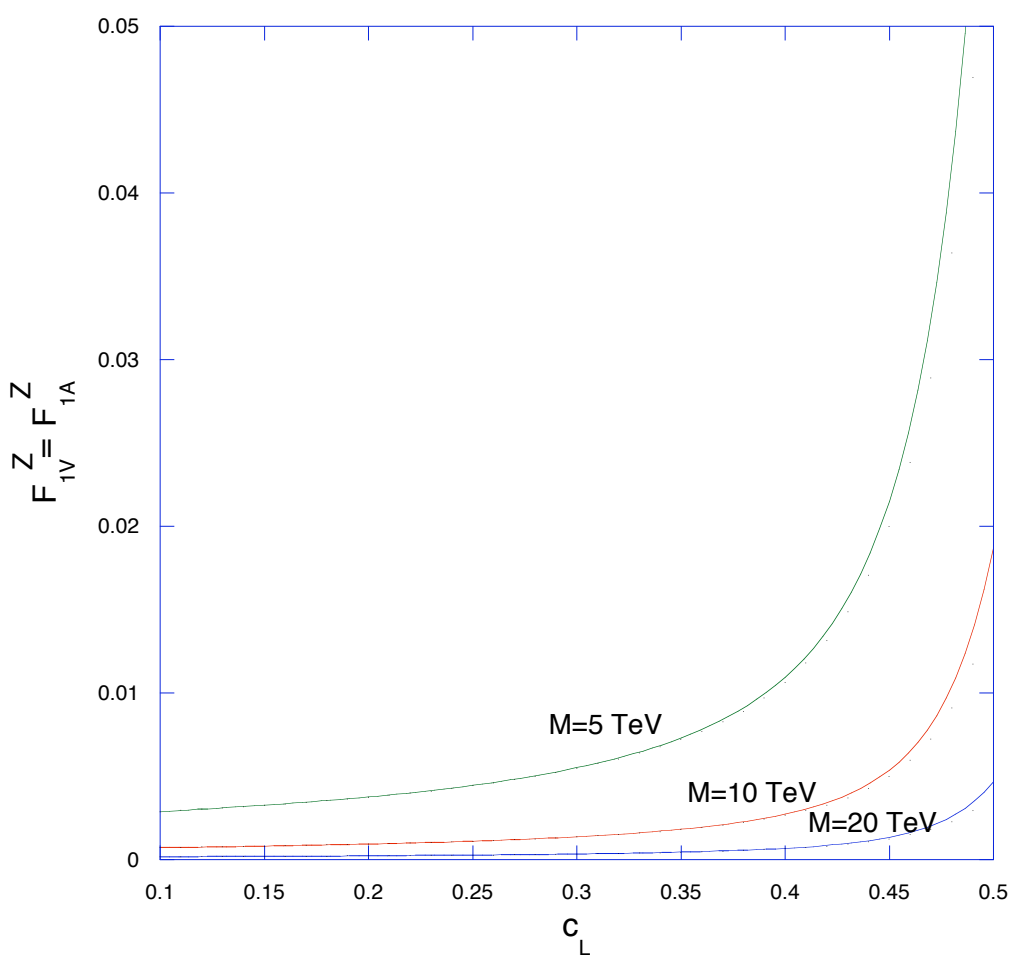

Figure 6: Effects on the $\mathrm{Z}$ form factors due to top/KK-top mixing as a function of $c_{L}$ for various values of the KK-mass. A high luminosity ILC should have a sensitivity of 0.006 to these form factors, and could optimistically reach 0.003 . 
and $F_{1 A}^{Z}$. Here, one looks for deviations in the right-handed top quark couplings, and this might require determination of the top quark polarization. Previous analyses have looked at $F_{1 V}^{Z}$ and $F_{1 A}^{Z}$ separately (assuming one is nonzero and all others vanish)-here a more unified analysis for the ILC would be welcomed.

Finally, we consider the effects of brane kinetic terms (BKTs). A detailed discussion of these terms in flat space can be found in Ref. 223. In the context of Randall-Sundrum models, two papers by Carena, Delgado, Ponton, Tait and Wagner (CDPTW) [16, 17] have extensively studied BKTs and their effects on phenomenology. The BKTs for fermions arise in the 5D action

$$
S=-\int d^{4} x \int_{0}^{\pi R} d y \sqrt{-G}\left(i \bar{\Psi} \Gamma^{A} e_{A}^{M} D_{M} \Psi+i m(y) \bar{\Psi} \Psi+2 \alpha_{f} \delta(y-\pi R) \bar{\Psi}_{L} \gamma^{a} e_{a}^{\mu} \partial_{\mu} \Psi_{L}\right)
$$

where $\Gamma$ and $\gamma$ are the $5 \mathrm{D}$ and $4 \mathrm{D}$ Dirac matrices, and the last term is the BKT. Here, the $\delta$ function is normalized so that $\int_{0}^{\pi R} 2 \delta(y) d y=1$. The coefficient, $\alpha_{f}$, has dimensions of length. Note that this is an IR-brane BKT, whereas a UV brane BKT would be proportional to $\delta(y)$, but one expects the UV brane BKTs to be less phenomenologically relevant. More details can be found in CDPTW.

One can also have gauge field BKTs. For a $U(1)$ gauge group, the relevant part of the action is

$$
S=-\int d^{4} x \int_{0}^{\pi R} d y \frac{1}{2} B_{\mu} \mathcal{O}^{\mu \nu} B_{\nu}
$$

where

$$
\mathcal{O}^{\mu \nu}=\frac{1}{g_{5}^{2}}\left(P^{\mu \nu}+\eta^{\mu \nu} \partial_{y}\left(e^{-2 \sigma} \partial_{y}\right)+2 \delta(y) r_{U V} P^{\mu \nu}+2 \delta(y-\pi R) r_{I R} P^{\mu \nu}\right)
$$

and $P^{\mu \nu} \equiv \eta^{\mu \nu} \partial^{2}-\partial^{\mu} \partial^{\nu}$. Note that we have explicitly included both UV and IR BKTs.

CDPTW [16, 17] use these actions and find all of the KK-masses, wavefunctions and couplings in the model, and the reader is referred to those papers for the full expressions. They find that the IR BKTs repel the KK-wavefunctions from the IR brane, thus reducing the couplings of the zero-mode fermions to the KK gauge bosons. As a result, the effects on precision tests 
is reduced, and KK-masses of the order of a few $\mathrm{TeV}$ (and thus in reach of the LHC) become allowed. In addition, BKTs can also make the model more compatible with grand unification. Relatively large BKTs (of order $\pi R$ ) are needed to have a substantial impact, but such terms are not unnatural.

As discussed in the introduction, our approach in this paper is to consider KK-masses which are out of reach of the LHC. The effect of the BKTs discussed by CDPTW is then to reduce the coupling of fermons to KK-gauge bosons, and thus lower the effects in top pair production. In short, we have added some parameters to the model which, if large enough, can substantially weaken our bounds .

One interesting feature concerns the conformal limit $\left(c_{L}=c_{R}=1 / 2\right)$. At this point, without BKTs, the coupling of the zero mode fermions to the KK-gauge bosons vanish, and all of the contributions we discussed (involving KK-gauge bosons) vanish (as well as many contributions to electroweak precision tests). This is because the fermion zero-mode wavefuntion is flat, and thus proportional to the gauge zero-mode wavefunction, which is orthogonal to the KKgauge boson wavefunctions. This was first noticed in the Randall-Sundrum model in Ref. [21, and for Higgsless models in Ref. 31]. With BKT's however, unless the gauge and fermion BKTs are identical, the fermion and gauge boson orthogonality conditions will differ, and the couplings won't vanish in the conformal limit. Whether the couplings are large enough to make a measureable contribution depends, of course, on the size of the BKTs.

\section{Discussion and Conclusions}

The Randall-Sundrum model is one of the most promising approaches to solving the gauge hierarchy problem. The five-dimensional spacetime compactified on an orbifold, with a slice of $\mathrm{ADS}_{5}$ describing the bulk geometry, can not only explain a large hierarchy but also may naturally arise from string theory. The original form of the model had all of the Standard Model particles on the $\mathrm{TeV}$ brane, but there has been much interest in versions of the model in 
which gauge bosons and/or fermions can propagate. Such models can also naturally explain the fermion mass hierarchy. In this case, the KK excitations of the gauge bosons and/or fermions can have significant phenomenological consequences.

Most analyses of the phenomenology of the Randall-Sundrum models have looked at the effects of the KK excitations on precision electroweak constraints, and there have been many interesting modifications to the model which ameliorate many of these constraints. This can allow the KK excitations to be within reach of the LHC. The most appealing of these modifications include imposing a custodial $S U(2)$ gauge symmetry in the bulk (which may come from a global $S U(2)$ symmetry in the AdS/CFT related conformal theory), or by adding gauge or fermion brane kinetic terms, or both.

Our approach is different. We will suppose that the KK excitations have masses well in excess of $5 \mathrm{TeV}$, and are thus out of range of the LHC. We also do not concern ourselves with precision electroweak constraints (which may still be signficant in the $5-15 \mathrm{TeV}$ mass range), assuming that one of the modifications discussed above can ameliorate the constraints if necessary. We have argued that top pair production could be the first signature of these excitations, since the top quark, due to its large mass, must be close to the TeV brane and thus will feel the effects of these excitations more strongly than other fermions.

We have calculated top pair production at the ILC in the Randall-Sundrum model. Note that in many versions of the model, such as the version with a custodial $S U(2)$ symmetry or versions with extended gauge or fermion sectors, there will be additional fields which could affect top pair production. Unless there is destructive interference plus some tuning, however, such fields are likely to increase the bounds. For simplicity, we have only considered the KKexcitations of standard model particles.

When all fermions are on the $\mathrm{TeV}$ brane, direct KK-gauge boson exchange gives a sensitivity to KK-gauge boson masses up to $150 \mathrm{TeV}$. The most attractive models, though, are those in which fermions propagate in the bulk. In this case, the tree-level KK-gauge boson exchange 
diagram still dominates for much of parameter-space, but the reach is much smaller, since the electron coupling is much weaker. We found the change in the cross-section and left-right asymmetry as a function of the fermion mass paramters and the KK-gauge boson mass, and obtained a sensitivity to KK-gauge boson masses of approximtely 10-20 TeV, depending on the mass parameters.

We then considered the one-loop diagrams in which KK-gauge bosons are exchanged by the top quarks in the final state. The dominant diagram is due to KK-gluon exchange. These will affect the $\gamma$ and $Z$ form factors, and we find sensitivity in much of parameter-space to $5 \mathrm{TeV}$ KK-gauge boson masses, but $10 \mathrm{TeV}$ masses are out of reach. The effects of KK-fermions on these results is small.

Finally, mixing between the top quarks and the KK-tops can be substantial in the narrow window in which $c_{L}$ is between 0.3 and 0.5 . Although this window is narrow, it is in the phenomenologically preferred range. The reach can exceed $10 \mathrm{TeV}$ for some of this range.

A more detailed phenomenological analysis is needed. Effects of positron polarization and top quark polarization have not really been included, the experimental sensitivities to the various form factors were determined by assuming that only one was nonzero, the relationship between those form factors and experimentally observed quantities is unclear (in view of different assumptions made). The basic version of the Randall-Sundrum model has only three parameters $-c_{L}, c_{R}$ and $M_{K K}$, with brane kinetic terms playing a role if they are sufficiently large. This is a sufficiently small parameter set that an event generator could be constructed. Recently, a version of Pythia for Universal Extra Dimensions 33. was developed; such a tool could be developed for this model. Certainly, one expects models with Kaluza-Klein excitations to behave in some sense like extra-Z models (as in tree-level exchange), and in some sense like anomalous gauge boson couplings (as in the one-loop diagrams and in mixing), so a Pythia-type gnerator would be helpful.

We are very grateful to Kaustubh Agashe, Chris Carone, Csaba Csaki, Josh Erlich, and 
Frank Petriello for extensive discussions. This work was initiated at the Aspen Center for Physics and was supported by the National Science Foundation grant PHY-023400. 


\section{APPENDIX}

The diagrams in Fig. 4 are calculated. The counterterms will be determined by the requirement that $\Gamma_{\mu}^{\gamma}\left(q^{2}=0\right)=-\frac{2}{3} i e_{R} \gamma_{\mu}$ and $\Gamma_{\mu}^{Z}\left(q^{2}=M_{Z}^{2}\right)=-\frac{g_{R}}{4 \cos \theta_{W R}} \gamma_{\mu}\left(1-\frac{8}{3} \sin ^{2} \theta_{W R}+\gamma_{5}\right)$.

We let the coupling of the gauge boson to the top quark be $C \gamma_{\mu}\left(1-\alpha \gamma_{5}\right)$. Note that the fact that the chiralities may have different enhancements implies that even the KK-gluon will not necessarily couple in a vector-like manner. The numerator of the massive vector propagator does contain a $k_{\mu} k_{\nu} / M^{2}$ term, but the divergences from this term are cancelled by the counterterms, and the finite parts are negligible. The corrections to the $\bar{t} t \gamma$ vertex due to the diagrams in Fig. 4 is given by

$$
\frac{i C^{2}}{16 \pi^{2}}\left(\frac{2}{3} e\right) \int_{0}^{1} d x \int_{0}^{1-x} d y\left(T_{A}^{\mu}\left(\frac{1}{\Delta_{0}}-\frac{1}{\Delta}\right)+\frac{T_{B}^{\mu}}{2} \ln \frac{\Delta_{0}}{\Delta}-\frac{q^{2} T_{q}^{\mu}}{\Delta}\right)
$$

where $\Delta_{0} \equiv M^{2}(1-x-y)+m^{2}(x+y), \Delta=-q^{2} x y+\Delta_{0}, M$ is the KK-gauge boson mass, $m$ is the top quark mass and

$$
\begin{aligned}
T_{A}^{\mu} & =2 m^{2}\left(-(x+y)^{2}\left(1+\alpha^{2}\right)+2(x+y)\left(-1+3 \alpha^{2}\right)+4\left(1-\alpha^{2}\right)\right) \gamma^{\mu} \\
& +4 \alpha m^{2}(x+y)(2-x-y) \gamma^{\mu} \gamma_{5} \\
& +2 m\left[(x+y)(x+y-1)+\alpha^{2}((x+y-1)(x+y-4))\right]\left(i \sigma^{\mu \nu} q_{\nu}\right) \\
T_{B}^{\mu} & =4\left(1+\alpha^{2}\right) \gamma_{\mu}-8 \alpha \gamma^{\mu} \gamma_{5} \\
T_{q}^{\mu} & =-2(x y-x-y+1)\left(1+\alpha^{2}\right) \gamma^{\mu}+4 \alpha(x y-x-y+1) \gamma_{\mu} \gamma_{5} .
\end{aligned}
$$

The corrections for the $\bar{t} t Z$ vertex are given by

$$
\frac{i C^{2}}{16 \pi^{2}}\left(\frac{g}{4 \cos \theta_{W}}\right) \int_{0}^{1} d x \int_{0}^{1-x} d y\left(Z_{A}^{\mu}\left(\frac{1}{\Delta_{M_{Z}}}-\frac{1}{\Delta}\right)+Z_{B}^{\mu} \ln \frac{\Delta_{M_{Z}}}{\Delta}+Z_{q}^{\mu}\left(\frac{M_{Z}^{2}}{\Delta_{M_{Z}}}-\frac{q^{2}}{\Delta}\right)\right)
$$

where $\Delta_{M_{Z}}=-M_{Z}^{2} x y+\Delta_{0}, M$ is the mass of the KK-gauge boson, and $m$ is the top mass and

$$
\begin{aligned}
Z_{A}^{\mu} & =m^{2}\left(4 \alpha(1+2 \alpha)+2(x+y)\left(A\left(1-3 \alpha^{2}\right)+2 \alpha\right)-(x+y)^{2} B_{1}\right) \gamma^{\mu} \\
& +m^{2}\left(4 \alpha A+8 y\left(1+\alpha^{2}-2 \alpha A\right)+(x+y)^{2} B_{2}\right) \gamma^{\mu} \gamma_{5}
\end{aligned}
$$




$$
\begin{aligned}
& +m\left(2\left(A\left(1+\alpha^{2}\right)-2 \alpha\right)+(x+y)\left(A\left(1+5 \alpha^{2}\right)-6 \alpha\right)+(x+y)^{2} B_{1}\right) i \sigma^{\mu \nu} q_{\nu} \\
Z_{B}^{\mu} & =2\left(A\left(1+\alpha^{2}\right)-2 \alpha+2 \alpha^{2} A \frac{m^{2}}{M^{2}}\right) \gamma^{\mu} \\
& +2\left(1+\alpha^{2}-2 \alpha A+2 \alpha^{2} \frac{m^{2}}{M^{2}}\right) \gamma^{\mu} \gamma_{5} \\
Z_{q}^{\mu} & =\left(\left(A\left(1+\alpha^{2}\right)-2 \alpha\right)(1-x-y)-x y B_{1}\right) \gamma^{\mu} \\
& +\left(\left(1+\alpha^{2}-2 \alpha A\right)(1-x-y)-x y B_{2}\right) \gamma^{\mu} \gamma_{5}
\end{aligned}
$$

where $A=1-\frac{8}{3} \sin ^{2} \theta_{W}, B_{1}=A\left(1+\alpha^{2}\right)-2 \alpha+4 \alpha^{2} A \frac{m^{2}}{M^{2}}$, and $B_{2}=1+\alpha^{2}-2 \alpha A+4 \alpha^{2} \frac{m^{2}}{M^{2}}$.

A diagram not shown in Fig. 4 is the vacuum polarization diagram, in which the photon or $\mathrm{Z}$ propagator goes into a top quark loop, and then back to a KK-B or KK- $W_{3}$. We have calculated the contribution of this diagram and found it to be substantially smaller than the diagrams considered.

There is also the diagram in Fig. 4 in which the internal lines are b-quarks and the charged KK-W boson is exchanged. This gives (assuming $V_{t b}=1$ )

$$
-\frac{1}{3} \frac{i e}{16 \pi^{2}}\left(\frac{g^{(1)}}{2 \sqrt{2}}\right) \int_{0}^{1} d x \int_{0}^{1-x} d y\left(W_{A}^{\mu}\left(\frac{1}{\Delta_{M_{i}}}-\frac{1}{\Delta_{W}}\right)+\frac{W_{B}^{\mu}}{2} \ln \frac{\Delta_{M_{i}}}{\Delta_{W}}+W_{q}^{\mu}\left(\frac{M_{i}^{2}}{\Delta_{M_{i}}}-\frac{q^{2}}{\Delta_{W}}\right)\right)
$$

where $\Delta_{W}=-q^{2} x y-m^{2}(x+y)(1-x-y)+m_{b}^{2}(x+y)+M^{2}(1-x-y), \Delta_{M_{i}}=-M_{i}^{2} x y-$ $m^{2}(x+y)(1-x-y)+m_{b}^{2}(x+y)+M^{2}(1-x-y), M$ is the mass of the KK-gauge boson and $m$ is the top quark mass as before, here $m_{b}$ is the mass of the bottom quark and

$$
\begin{aligned}
W_{A}^{\mu} & =-2\left(m^{2}(1-a)(1-x-y)^{2}+m_{b}^{2}(1+a)\right) \gamma^{\mu} \\
& -2\left(m^{2}(1-a)(1+x+y)^{2}-m_{b}^{2}(1+a)\right) \gamma^{\mu} \gamma_{5} \\
& +2 m(1-a)\left(2-3(x+y)+(x+y)^{2}\right) i \sigma^{\mu \nu} q_{\nu} \\
W_{B}^{\mu} & =4(1-a) \gamma^{\mu}-4(1-a) \gamma^{\mu} \gamma_{5} \\
W_{q}^{\mu} & =-2(1-a)(1-x-y+x y) \gamma^{\mu}+2(1-a)(1-x-y+x y) \gamma^{\mu} \gamma_{5}
\end{aligned}
$$

For $\bar{t} t \gamma, M_{i}=0$ and $a=0$ For the $\bar{t} t Z, M_{i}=M_{Z}$ and $a=\frac{1}{\frac{4}{3} \sin ^{2} \theta_{W}}$.

Finally, there is one diagram that we have not discussed. The $\gamma$ or $Z$ can convert into 
a pair of charged $K K-W$-bosons, which then exchange a b-quark and convert into a top pair. As noted earlier, the diagram in Figure 4 for KK-gluon exchange completely dominates the results, and the finite contribution of this " 2 -W" diagram is negligible. However, here the divergences are not removed by the counterterms. This should not be surprising. We have used the gauge choice in which $A_{5}=0$. This is the unitary gauge, and is problematic for evaluating loop diagrams, since the gauge boson propagtors have bad high-energy behavior (the finite Smatrix only results from cancellations among divergent Green's functions). This has not been a problem for the other diagrams. In this case, one should use another gauge, such as the ' $t$ Hooft-Feynman gauge, and include the higher modes of the $A_{5}$ field. Since the finite part of the diagram is so much smaller than that from the KK-gluon exchange (due to a much weaker coupling and two heavy fields in the loop rather than one), we will not include this diagram. A nice discussion can be found in Ref. [32].

\section{References}

[1] L. Randall and R. Sundrum, Phys. Rev. Lett. 83, 3370 (1999) arXiv:hep-ph/9905221.

[2] C. Csaki, arXiv:hep-ph/0404096, T. G. Rizzo, eConf C040802, L013 (2004) arXiv:hep-ph/0409309; C. Csaki, arXiv:hep-ph/0404096.

[3] H. Davoudiasl, J. L. Hewett and T. G. Rizzo, Phys. Rev. Lett. 84, 2080 (2000) arXiv:hep-ph/9909255.

[4] A. Pomarol, Phys. Lett. B 486, 153 (2000) arXiv:hep-ph/9911294.

[5] H. Davoudiasl, J. L. Hewett and T. G. Rizzo, Phys. Lett. B 473, 43 (2000) arXiv:hep-ph/9911262.

[6] S. Chang, J. Hisano, H. Nakano, N. Okada and M. Yamaguchi, Phys. Rev. D 62, 084025 (2000) arXiv:hep-ph/9912498. 
[7] S. J. Huber and Q. Shafi, Phys. Rev. D 63, 045010 (2001) arXiv:hep-ph/0005286.

[8] C. Csaki, J. Erlich and J. Terning, Phys. Rev. D 66, 064021 (2002) arXiv:hep-ph/0203034.

[9] H. Georgi, A. K. Grant and G. Hailu, Phys. Lett. B 506, 207 (2001) arXiv:hep-ph/0012379.

[10] M. Carena, T. M. P. Tait and C. E. M. Wagner, Acta Phys. Polon. B 33, 2355 (2002) arXiv:hep-ph/0207056.

[11] H. Davoudiasl, J. L. Hewett and T. G. Rizzo, Phys. Rev. D 68, 045002 (2003) arXiv:hep-ph/0212279.

[12] T. Gherghetta and A. Pomarol, Nucl. Phys. B 586, 141 (2000) arXiv:hep-ph/0003129.

[13] D. Dooling and K. Kang, Phys. Lett. B 502, 189 (2001) arXiv:hep-ph/0009307.

[14] H. Davoudiasl, J. L. Hewett and T. G. Rizzo, Phys. Rev. D 63, 075004 (2001) arXiv:hep-ph/0006041.

[15] S. J. Huber, C. A. Lee and Q. Shafi, Phys. Lett. B 531, 112 (2002) arXiv:hep-ph/0111465.

[16] M. Carena, A. Delgado, E. Ponton, T. M. P. Tait and C. E. M. Wagner, Phys. Rev. D 68, 035010 (2003) arXiv:hep-ph/0305188.

[17] M. Carena, A. Delgado, E. Ponton, T. M. P. Tait and C. E. M. Wagner, Phys. Rev. D 71, 015010 (2005) arXiv:hep-ph/0410344.

[18] Y. Grossman and M. Neubert, Phys. Lett. B 474, 361 (2000) arXiv:hep-ph/9912408.

[19] S. J. Huber and Q. Shafi, Phys. Lett. B 498, 256 (2001) arXiv:hep-ph/0010195.

[20] S. J. Huber, Nucl. Phys. B 666, 269 (2003) arXiv:hep-ph/0303183. 
[21] K. Agashe, A. Delgado, M. J. May and R. Sundrum, JHEP 0308, 050 (2003) arXiv:hep-ph/0308036.

[22] J. L. Hewett, F. J. Petriello and T. G. Rizzo, JHEP 0209, 030 (2002) arXiv:hep-ph/0203091.

[23] F. del Aguila, M. Perez-Victoria and J. Santiago, Acta Phys. Polon. B 34, 5511 (2003) arXiv:hep-ph/0310353 ; F. del Aguila, M. Perez-Victoria and J. Santiago, Eur. Phys. J. C 33, S773 (2004) arXiv:hep-ph/0310352; F. del Aguila, M. Perez-Victoria and J. Santiago, JHEP 0302, 051 (2003) arXiv:hep-th/0302023.

[24] G. Moreau and J. I. Silva-Marcos, JHEP 0603, 090 (2006) arXiv:hep-ph/0602155.

[25] T. Appelquist, H. C. Cheng and B. A. Dobrescu, Phys. Rev. D 64, 035002 (2001) arXiv:hep-ph/0012100.

[26] S. Godfrey, eConf C960625, NEW138 (1996) arXiv:hep-ph/9612384.

[27] T. Abe et al. [American Linear Collider Working Group], in Proc. of the APS/DPF/DPB Summer Study on the Future of Particle Physics (Snowmass 2001) ed. N. Graf, arXiv:hep-ex/0106057.

[28] U. Baur, arXiv:hep-ph/0508151

[29] J. A. Aguilar-Saavedra et al. [ECFA/DESY LC Physics Working Group], arXiv:hep-ph/0106315.

[30] A. Juste et al., "Report of the 2005 Snowmass top / QCD working group," arXiv:hep-ph/0601112.

[31] G. Cacciapaglia, C. Csaki, C. Grojean and J. Terning, Phys. Rev. D 71, 035015 (2005) arXiv:hep-ph/0409126. 
[32] L. Randall and M. D. Schwartz, JHEP 0111, 003 (2001) arXiv:hep-th/0108114; L. Randall and M. D. Schwartz, Phys. Rev. Lett. 88, 081801 (2002) arXiv:hep-th/0108115; K. Agashe, A. Delgado and R. Sundrum, Nucl. Phys. B 643, 172 (2002) arXiv:hep-ph/0206099.

[33] B. C. Allanach et al., "Les Houches 'Physics at TeV colliders 2005' Beyond the standard model working group: Summary report," arXiv:hep-ph/0602198. 